\title{
Shading Approach for Artistic Stroke Thickness using 2D Light Position
}

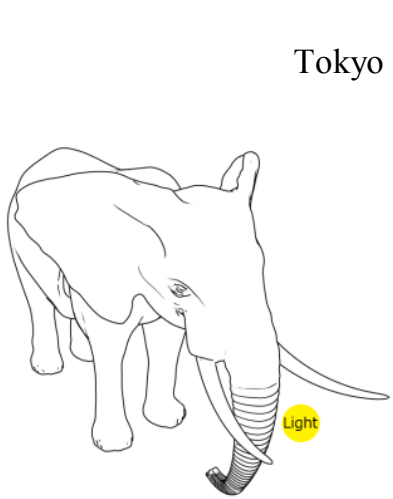

(a)

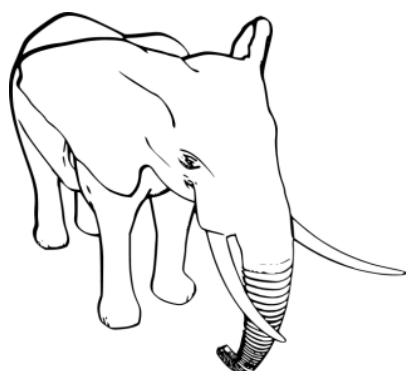

(b)

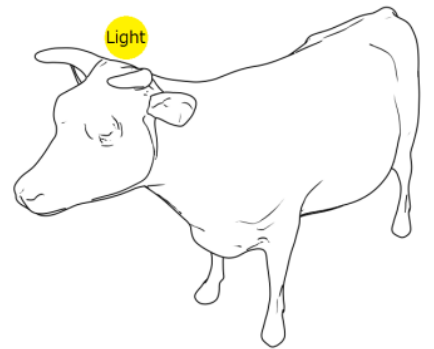

(c)

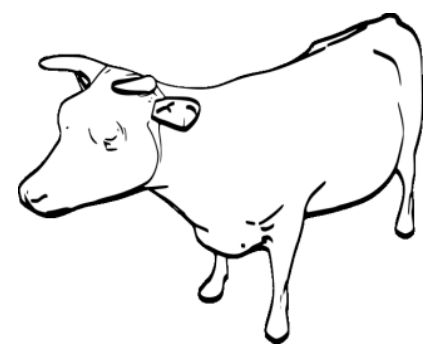

(d)

Figure 1: (a) Input image of an elephant and light position; (b) our result using (a); (c) input image of cattle and light position; (d) Our result using (c).

\section{Introduction}

We present an approach for determining stroke thickness in illustrations based on two-dimensional (2D) light position. Stroke rendering is particularly important for cartoons and illustrations in nonphotorealistic rendering (NPR). In cartoons and illustrations, stroke thickness is varied for effective expressions.

[Goodwin et al. 2007] proposed a shading approach to artistic stroke thickness using a three-dimensional (3D) model. However, shading 2D images using 2D light position remains a challenge.

We propose a method to render artistic stroke styles by varying thickness considering the distance from a 2D light position.

\section{Our Approach}

Interface The proposed method performs illustration styled shading in real-time by specifying the position of the light in an input image according to the mouse pointer position (Figures 1a, 1c).

Summary Our shading approach is based on an assumption presented by [Goodwin et al. 2007]: dark strokes are drawn to approximate dark regions of the shaded parts. First, the user inputs a bitmap image of constant thickness strokes. Then, the input image is segmented into black closed areas to express a wide range of stroke thicknesses at each area. Each area is shaded so that the thickness changes partially by considering the 2D light position. Finally, strokes are rendered in an illustration style by synthesizing the thickened lines. We use [Potrace] to vectorize our result.
Segmentation of input bitmap strokes The input image is first transformed to a binary image (black or white). Then, sets of pixels of consecutive white areas are extracted by a labeling process with their four neighboring black pixels as the segmented areas. These black pixels are defined as stroke pixels.

We describe our thickening process using the 2D light position mentioned below.

Calculation of shading direction First, light directions are calculated for all stroke pixels. Then, the light directions are approximated for eight directions. Finally, the opposite direction of the light direction is obtained as the shading direction.

Calculation of line width Here, the strokes are thickened to the shading direction. In each stroke pixel, the thickening width $W_{i}$ (pixels) is determined by considering the 2D Euclidean distance from the light position $D_{i}$ where $i=0,1 \ldots n ; n$ is the number of stroke pixels. The user specifies the maximum value of the line width $W_{\text {max }}$. In each area, the maximum value of the distance $D_{i}$ is $D_{\max }$. The width $W_{i}$ is calculated by the following equation.

$$
W_{i}=\frac{D_{i} W_{\max }}{D_{\max }}
$$

Then, we thicken strokes by changing sequential $W_{i}$ pixels in the shading direction of each stroke pixels into stroke pixels.

\section{Result}

Figure 1 shows input images (cattle and elephant) and our results. We administered a questionnaire to compare the results of [Goodwin] with the results of the proposed method (see supplemental material). The results of the questionnaire indicate that our method can render artistic strokes from 2D image and light position as well as the 3D method.

\section{References}

Goodwin, T., Vollick, I., And Hertwmanj, A. 2007. Isophote Distance: A Shading Approach to Artistic Stroke Thickness. In Proc. of NPAR, 53-62.

POTRACE http://potrace.sourceforge.net
Permission to make digital or hard copies of part or all of this work for personal or classroom use is granted without fee provided that copies are not made or distributed for commercial advantage and that copies bear this notice and the full citation on the first page. Copyrights for third-party components of this work must be honored. For all other uses, contact the Owner/Author.

SIGGRAPH 2014, August 10 - 14, 2014, Vancouver, British Columbia, Canada.

2014 Copyright held by the Owner/Author.

ACM 978-1-4503-2958-3/14/08 